\title{
The Impact of Financial Crisis of 2007-2008 on Crude Oil Price
}

\author{
Xun Zhang, Lean Yu, and Shouyang Wang* \\ Institute of Systems Science, Academy of Mathematics and Systems Science, \\ Chinese Academy of Sciences, Beijing 100190, China \\ \{zhangxun, leanyu, sywang\} @amss.ac.cn
}

\begin{abstract}
For better estimation of the impact of extreme events on crude oil price volatility, an EMD-based event analysis approach is proposed. In this method, the time series to be analyzed is first decomposed into several intrinsic modes with different time scales from fine-to-coarse and an average trend. The decomposed modes respectively capture the fluctuations caused by the extreme event or other factors during the analyzed period. The total impact of an extreme event is included in only one or several dominant modes, but other modes provide valuable information for subsequent factors. The effects of financial crisis of 2007-2008 to crude oil price are analyzed through this method and empirical results reveal that the EMD-based event analysis method provides a feasible solution to estimating the impact of extreme events on crude oil prices.
\end{abstract}

Keywords: Crude Oil Price, Financial Crisis, Event Analysis, Empirical Mode Decomposition.

\section{Introduction}

The financial crisis that began on 9 August 2007 ranks among the most serious economic events affecting the global economy since the Great Depression of the 1930s. The crisis has also affected the oil market and contributed to the collapse of oil prices in 2008. The closing price of West Texas Intermediate crude oil price, which rose to an all-time high of $\$ 145.31$ per barrel on 3 July 2008, was driven down to $\$ 41$ per barrel on December 5, 2008, a drop of $72 \%$ in five months, mainly due to the slowdown in economic growth in the United States and other parts of the world. In fact, the variations of crude oil price are strongly related to extreme events such as wars and economic recessions [1][2]. Some literatures have spawned to utilize the effects of historical extreme events in crude oil price modeling and forecasting. For example, TEI@I methodology, a systematic crude oil price forecasting methods incorporating the effects of extreme events have been constructed and its empirical results have shown its superiority [3][4]. Others methods based on historical information of events include rough-set refining text mining, pattern matching methods and so on [5][6].

${ }^{*}$ Corresponding author. Tel.: 86-10-62651375, Fax: 86-10-62621324 
However, the fundamental of incorporating historical events in forecasting is to analyze the effects of events. Traditional event evaluation methods mainly include two kinds: intervention analysis and event study approach. Intervention analysis is one of the most rigorous statistical modeling techniques, which is used to test whether a postulated event caused a change in the time series and, if so, what are the magnitude and the nature of the change [7][8]. This method is only applicable to linear and stationary time series because essentially it is based on linear time series model. Likewise, the event study method has been a standard analysis tool for assessing the financial or economic impact of specific unanticipated events in economics, accounting and finance [9]. It assumes the efficient market hypothesis (EMH) must be satisfied. But whether the EMH holds for crude oil markets is an open question.

In summary, both the two traditional methods consider a time series as the sum of a normal evolution part and an exceptional shock, and then they implement the "divide and conquer" strategy to separately modeling the two components. But as addressed by Huang et al. [10][11], although a complex time series can be treated as sums of several simple oscillation patterns, generally the number of these patterns is more than two. Therefore, a feasible decomposition method which can extract the inherent oscillations from the time series could provide more information for event evaluation. Therefore, a competitive decomposition algorithm - empirical mode decomposition (EMD), is applied to study the behaviors of crude oil price during the recent financial crisis.

In the innovative EMD-based event analysis approach, the original time series is first decomposed into some independent oscillations, and then the concrete implications of each oscillation are identified. Usually, the dominant oscillations are purely caused by the extreme event of interest and they are used to approximate the pattern and magnitude of the change. At the same time, other oscillations provide useful information about changes triggered by the event at different time scales, such as the effects of the event to short-term fluctuations and the long term trend. The EMDbased method has some advantages over the intervention analysis and event study approach. Firstly, it is suitable for nonlinear and non-stationary data. Secondly, it provides a multiscale framework to analyze the impact of the extreme event, including both direct impact and indirect impact.

The rest of this study is organized as follows. Section 2 briefly describes the EMD algorithm and the EMD-based event analysis approach. The effects of financial crisis to crude oil price are evaluated through this method in Section 3. In Section 4, Concluding remarks are given.

\section{Methodology Formulation}

\subsection{Empirical Mode Decomposition}

Empirical mode decomposition (EMD) is a promising nonlinear, non-stationary data processing method proposed by Huang et al. (1998) [10]. It considers the real time series as fast oscillations superimposed on slow oscillations. Those oscillations are approximated by "intrinsic mode functions" (here after IMF, or mode). An IMF must satisfy the following two conditions: 1) the numbers of extrema and zero-crossings 
are the same, or differ at the most by one; 2) they are symmetric with respect to local zero mean.

A sifting process is designed to extract IMFs level by level. First, the IMF with the highest frequency riding on the lower frequency part of the data is extracted, and then the IMF with the next highest frequency is extracted from the differences between the data and the extracted IMF. The iterations continue until no IMF is contained in the residual. The overall sifting procedure for a time series $\mathrm{x}(\mathrm{t})$ is described as follows.

1) Initialize: set $r_{0}(t)=x(t), i=$;

2) Extract the $i^{\text {th }} \mathrm{IMF}$ :

2.1) Initialize: set $d_{0}(t)=r_{i-1}(t), k=1$;

2.2) Identify all the maxima and minima of $d_{k-1}(t)$;

2.3) Generate the upper and lower envelopes, $e_{\min }(t)$ and $e_{\max }(t)$, of $d_{k-1}(t)$, with cubic spline interpolation.

2.4) Calculate the point-by-point mean, $m(t)$, from upper and lower envelopes:

$$
m(t)=\left(e_{\min }(t)+e_{\max }(t)\right) / 2
$$

2.5) Extract the mean from the $d_{k-1}(t)$ and define the difference of $d_{k-1}(t)$ and $m(t)$ as $d_{k}(t)$ :

$$
d_{k}(t)=d_{k-1}(t)-m(t)
$$

2.6) Check the properties of $d_{k}(t)$. If it is an IMF, denote $d_{k}(t)$ as the $i^{t h} \mathrm{IMF}: c_{i}(t)$; else, set $k=k+1$ and go back to 2.2$)$;

3) Define $r_{i+l}(t)=r_{i}(t)-c_{i}(t)$;

4) Check whether the following two stopping criteria are satisfied: (1) the number of extrema included in $r_{i+1}(t)$ is smaller than 3; (2) the amplitudes of $r_{i+l}(t)$ are far smaller than the amplitudes of $r_{i}(t)$ at each point.

If the stopping criteria are not satisfied, set $i=i+1$ and go back to 2) to extract another IMF; else the sifting process is completed, and the final $r_{i+1}(t)$ is the average trend of $x(t) . x(t)$ can be represented as the sum of IMFs with the residue.

In practice, it is an improvement of EMD, ensemble EMD (EEMD) method, are widely used [12][13]. The EEMD is designed to overcome the mode mixing problem when intermittency exists in data.

\subsection{EMD-Based Event Analysis}

The overall process of applying EMD to event analysis is described as follows:

Step1: Determining data frequency and analysis window. The first step is selecting analyzed data according to the event of interest. Then the data is divided into two sub periods: estimation window and event window. The estimation window is defined as periods without the effects of the event, and the event window as periods that include the effects of the event.

Step2: Decomposing data by EMD. After the preliminary understanding of the event and identification of data, the time series is decomposed into several IMFs. 
For data with intermittencies, EEMD is better than EMD for its ability to avoid the scale mixing.

Step3: Analyzing intrinsic modes. The first task in this step is to find the mode which sketches the overall change made by the extreme event in the analysis window. As mentioned before, each IMF has a concrete implication, representing a meaningful component of the original time series. Generally the effect of the extreme event is represented by one or sum of a few IMFs. This IMF or the sum of these IMF is treated as a main mode of the time series. Since the noises and long term trends contained in original time series are cleared away, the main mode gives a clear evaluation for the pattern and magnitude of change made by the extreme event in the event window. However, effects of some events, which themselves contain superimposed signals belonging to different scales, may be decomposed into several IMFs. In such a situation, those IMFs should be summed up into a component. Summing up more than one IMF is called "composition".

Besides the main mode, the event may also influence analyzed time series in other scales. For example, uncertainties brought by the event often make the analyzed indicator exhibit quick and small fluctuations. This can be tested by comparing the energy (square of the amplitude) of the IMF. Furthermore, the Hilbert spectrum can tell the differences in energy-frequency distribution between the event and estimation windows.

Finally, conclusions are drawn on the basis of analysis in accordance with steps 1) to 3). The pattern and magnitude of change made by the event are summarized. Then economic explanations are given.

\section{Estimating the Effect of 2008 Financial Crisis on Crude Oil Price Volatility}

\subsection{The Data}

The price series provided in this section include two benchmark daily time series for crude oil: West Texas Intermediate spot price (WTI) and European Brent spot price (Brent). The time period is divided into two sub periods: March 24, 2006 - August 8, 2007 as the estimation window and August 9, 2007 - December 16, 2008 as the event window. Each sub period contains 342 data points. All data are from Energy Information Administration, United States. We used the date as the dividing time line for these two periods when the large French bank BNP Paribas temporarily halted redemptions from three of its funds. Although a complete chronology of the crisis might start in February 2007 when several large subprime mortgage lenders started to report losses. The August 9, 2007 is recognized as the real trigger [14].

\subsection{Decomposition by EEMD}

EEMD is applied to decompose both WTI and Brent crude oil prices. An ensemble with 100 members is used, and the white noises added in each ensemble member are generated randomly from a normal distribution with standard deviation of 0.2. The stopping criterion for each sifting process is the same as that described in Section 2.1. Both the data series are decomposed into 8 IMFs plus 1 residue. Fig. 1 is the visualization. 


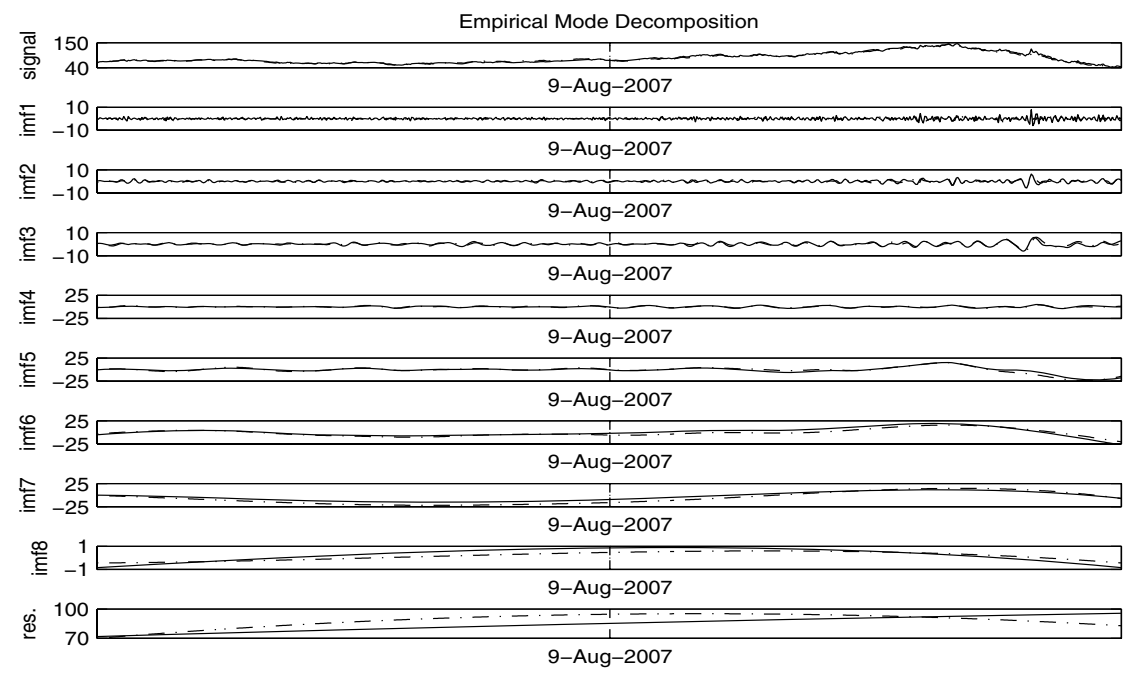

Fig. 1. The IMFs and residue for the WTI (solid line) and Brent (dotted line) crude oil prices. The first panel is the visualization of original time series.

In order to understand the features of price volatilities in different time scales, the values of IMFs in the estimation and event window are individually statistically analyzed. Three kinds of measures are calculated: mean period, correlation coefficients, and variance percentage of all the IMFs. Since the frequency and the amplitude of an IMF may change continuously with time, the periods are not constant. The mean period is defined as the value derived by dividing the total number of points by the number of peaks for each IMF. Correlation coefficients are used to measure the relationship between the individual component and the original time series. Variances of each IMF's percent of all IMFs are used to explain the contribution of each IMF to the total volatility of the observed data. The statistical measures are presented in Table 1 and Table 2.

Table 1. Measures of IMFs and the residue for the WTI and Brent daily crude oil price in the analysis window

\begin{tabular}{cccc}
\hline & \multicolumn{3}{c}{ WTI / Brent (analysis window) } \\
\hline & Mean Period & Correlation Coefficient & Variance percentage \\
IMF1 & $2.9 / 3.0$ & $0.07 / 0.06$ & $0.84 / 0.43$ \\
IMF2 & $6.6 / 6.3$ & $0.08 / 0.06$ & $0.57 / 0.39$ \\
IMF3 & $12.7 / 14.3$ & $0.17 / 0.09$ & $1.09 / 0.95$ \\
IMF4 & $34.2 / 34.2$ & $0.08 / 0.18$ & $1.66 / 1.09$ \\
IMF5 & $114 / 114$ & $0.85 / 0.80$ & $31.50 / 32.25$ \\
IMF6 & $171 / 171$ & $0.96 / 0.95$ & $43.61 / 25.83$ \\
IMF7 & $342 / 342$ & $0.81 / 0.68$ & $17.02 / 34.20$ \\
IMF8 & $342 / 342$ & $0.24 / 0.46$ & $0.11 / 0.03$ \\
Residue & & $0.08 / 0.30$ & $3.60 / 4.84$ \\
\hline
\end{tabular}


Table 2. Measures of IMFs and the residue for the WTI and Brent daily crude oil price in the event window

\begin{tabular}{cccc}
\hline & \multicolumn{3}{c}{ WTI / Brent (event window) } \\
\hline & Mean Period & Correlation Coefficient & Variance percentage \\
IMF1 & $2.8 / 2.8$ & $0.08 / 0.09$ & $0.76 / 0.35$ \\
IMF2 & $6.6 / 6.2$ & $0.10 / 0.11$ & $0.49 / 0.23$ \\
IMF3 & $13.2 / 12.2$ & $0.20 / 0.16$ & $0.85 / 0.39$ \\
IMF4 & $24.4 / 28.5$ & $0.34 / 0.37$ & $1.64 / 1.32$ \\
IMF5 & $57 / 68.4$ & $0.41 / 0.52$ & $4.30 / 4.58$ \\
IMF6 & $342 / 171$ & $0.80 / 0.66$ & $24.50 / 17.07$ \\
IMF7 & & $0.66 / 0.46$ & $40.89 / 33.36$ \\
IMF8 & & $-0.33 / 0.02$ & $0.46 / 0.07$ \\
Residue & & $-0.25 /-0.14$ & $26.13 / 42.65$ \\
\hline
\end{tabular}

It can be seen that there is no significant difference between the two price series except for the residue in event window. The high frequency IMFs, including IMF1 to IMF3, represent small fluctuations and exhibit similar behavior in both the two sub periods. They not only exhibit very low correlation coefficients with the observed data but also account for less than $1.1 \%$ of total variance. This means that these IMFs do not have serious effect on crude oil price. IMF1 captures the fluctuations with a cycle of 3 days, that is, the impact which is eliminated after 3 days on average. Similarly, IMF2 captures fluctuations over 1 week and IMF3 captures fluctuations over 2 and a half week. All the amplitudes of high frequency IMFs are less than $\$ 8$, which means high frequency fluctuations in the price series are no larger than $\$ 8$.

The impact of this financial crisis on crude oil price mainly exerts on low frequency IMFs. For IMF4 and IMF5, the mean periods in event window are shorter than those in the estimation window. For IMF4 to IMF6, the amplitudes in event window are much higher than those in the estimation window.

The IMF6 and IMF7 are two dominant modes during the whole time range. Variances of IMF6 and IMF7 rank highly among all the IMFs. Correlation coefficients between the two IMFs and the price series also reach a high level. The IMF8 and residue satisfy the trend definition given by [15]. Therefore they are essentially the long-term trend.

\subsection{Analyzing the Modes}

Dominant mode: the pattern of major impacts. Since the IMF6 and IMF7 are identified as dominant mode during the event window, they are used to outline the pattern of major impact of the crisis. The sum of the IMF6 and IMF7 in event window are normalized to $[0,1]$ and plotted in Fig.2. The dominant modes for WTI and Brent, almost perfectly match the shape of the original time series. We use this distance to measure the total impact of the financial crisis on crude oil prices. More specifically, by computing the distance of the local maximum in July 2008 and local minimum in the last day of the event window, we got the conclusion that the financial crisis have driven the WTI price downward $\$ 65$ and Brent price downward $\$ 63$ until December 16, 2008. By analyzing the dominant mode, of which the cycle does consist with the event window, rather than the original prices, the pattern and magnitude of the impact are very clear. 


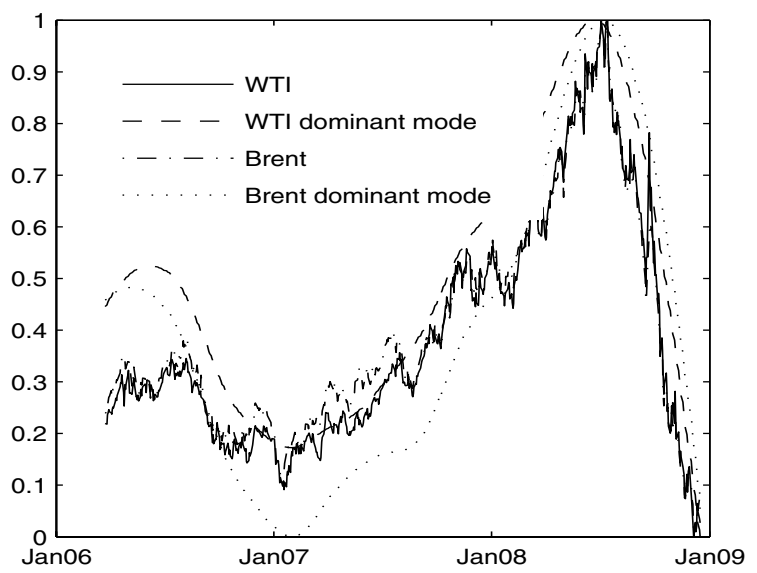

Fig. 2. The normalized prices and dominant mode derived from EEMD

High frequency IMFs: the total effect is near zero. The most high frequency mode, IMF1, captures most of the large and sudden jumps of the prices. The large changes of price series correspond to large amplitudes of IMF1. This means the period of the large jump is very short: a sharp rise (descent) always follows a sharp descent (rise) quickly. Thus the markets have the mean-reversion trend in the short-term.

However, in the whole event window, the total effects of these high frequency IMFs are near zero. This can be proved by a fine-to-coarse reconstruction. That is, using $t$-test to identify from which IMF $c_{i}$ the mean of the sum of $c_{1}$ to $c_{i}$ significantly departs from zero (null hypothesis). It shows that the mean of the reconstruction from IMF1 to IMF4 does not significantly depart from zero but the reconstruction from IMF1 to IMF5 does. Therefore, the sum of IMF1 to IMF4 does not have any long term effects on the prices and can be neglected if only the total impact of the event on the price series is of concern. This is another reason why we can take only IMF6 and IMF7 as the dominant IMFs representing the main change of prices during the event window.

The Spectrum analysis: the crisis amplifies the volatilities. In this analysis, we try to find whether the financial crisis amplified the crude oil price volatilities. Two methods, Hilbert spectrum analysis and t-test method are used. The EMD method, combined with Hilbert transform, is called Hilbert-Huang transform (HHT) since it is proposed by Huang et al. (1998). It presents the data clearly in a time-frequencyenergy space. Fig. 3 shows the Hilbert spectrum of WTI and Brent crude oil prices. The vertical axis is the normalized instantaneous frequency and the grayscale of the contours represents the energy.

Note that at each time point, the energy is mainly distributed at low frequency. It is not strange since the dominant mode is the low frequency IMF6 and IMF7. Looking at the high frequency region, the energy clusters in the event windows are slightly denser than those in the estimation window. At the same time, the energy in the event windows is higher than that in the estimation window To visualize it more clearly, we 

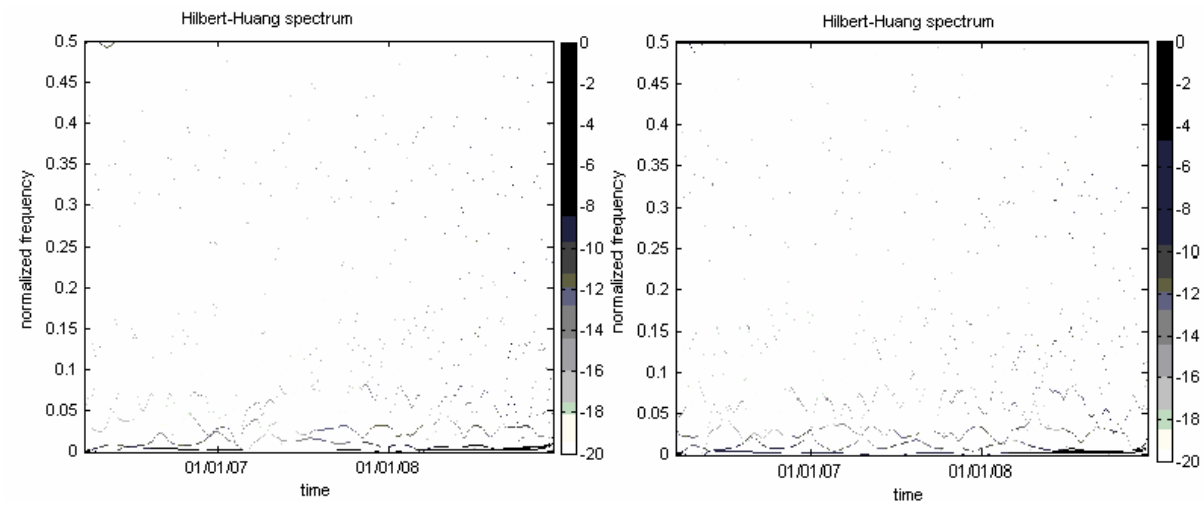

Fig. 3. The Hilbert spectrum of IMFs (left: WTI; right: Brent)

calculate the marginal energy density at time $t$, in normalized instantaneous frequency range $[0.6,1]$. Intuitive results are shown in Fig. 4 . It is very clear that the energy in the event window is much higher than that in the estimation window, especially the time the price drop sharply.

Then a t-test is implemented to test whether the amplitudes of IMF1 in the event window are significantly larger than those in the estimation window. The result does support the hypothesis. Therefore, we get the conclusion that the crisis actually increases the volatility of the crude oil markets in the event window.

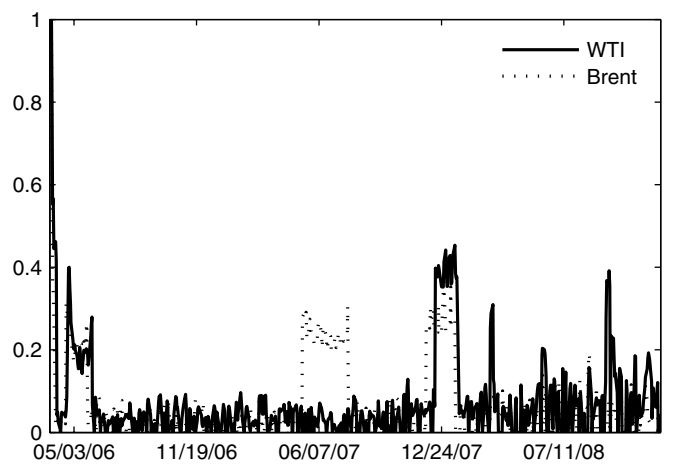

Fig. 4. The normalized prices and dominant mode derived from EEMD

\section{Conclusions}

In this paper, we first present an EMD-based event analysis method to estimate the impact of extreme events on crude oil price volatility, and then the effects of financial crisis of 2007-2008 to crude oil price are analyzed. The characteristics of the crisis to crude oil price can be summarized as below: 
The crisis had several types of impacts on crude oil prices: (i) high frequency fluctuations with small amplitudes; (ii) a large shock as a delta impulse, the time span of which is consistent with the event.

The sum of small fluctuations is near zero and does not have a long-term effect on crude oil prices. Analysts who address only the major impact of the event could ignore these high frequency fluctuations. But the volatilities of prices are increased during the financial crisis. This finding is similar with Ferderer (1996), which discovers the recessions of economic activities increase oil price volatility.

The large shock caused by the financial crisis, is represented by low frequency IMFs with long mean period and accounts for most of the variations in the event window. Through the numerical calculation, the financial crisis have driven the crude oil price downward more than $\$ 60$ until December, 2008, roughly $40 \%$ of the highest price, except for the effects of speculative activities and other impact factors.

Acknowledgments. This work is partially supported by grants from the National Natural Science Foundation of China (NSFC No. 70601029, 70221001).

\section{References}

1. Yang, C.W., Hwang, M.J., Huang, B.N.: An analysis of factors affecting price volatility of the US oil market. Energy Economics 24(2), 107-119 (2002)

2. Zhang, X., Lai, K.K., Wang, S.Y.: A new Approach for Crude Oil Price Analysis Based on Empirical Mode Decomposition. Energy Economics 30(3), 905-918 (2008)

3. Wang, S.Y., Yu, L., Lai, K.K.: Crude oil price forecasting with TEI@I methodology. Journal of Systems Sciences and Complexity 18(2), 145-166 (2005)

4. Yu, L., Wang, S.Y., Lai, K.K.: Forecasting Foreign Exchange Rates and International Crude Oil Price Volatility — TEI@I Methodology. Hunan University Press, Changsha (2007)

5. Yu, L., Wang, S.Y., Lai, K.K.: A Rough-Set-Refined Text Mining Approach for Crude Oil Market Tendency Forecasting. International Journal of Knowledge and Systems Sciences 2(1), 33-46 (2005)

6. Fan, Y., Liang, Q., Wei, Y.M.: A Generalized Pattern Matching Approach for Multi-Step Prediction of Crude Oil Price. Energy Economics 30(3), 889-904 (2008)

7. Box, G.E.P., Tiao, G.C.: Intervention Analysis with Applications to Economic and Environmental Problems. Journal of the American Statistical Association 70, 70-79 (1975)

8. Bonham, C.S., Gangnes, B.: Intervention Analysis with Cointegrated Time Series: The Case of the Hawaii Hotel Room Tax. Applied Economics 28(10), 1281-1293 (1996)

9. Mackinlay, A.C.: Event Studies in Economics and Finance. Journal of Economic Literature 35, 13-39 (1997)

10. Huang, N.E., Shen, Z., Long, S.R., Wu, M.C., Shih, H.H., Zheng, Q., Yen, N.-C., Tung, C.C., Liu, H.H.: The Empirical Mode Decomposition and the Hilbert Spectrum for Nonlinear and Non-Stationary Time Series Analysis. Proceedings of Royal Society of London 454, 903-995 (1998)

11. Rilling, G., Flandrin, P., Goncalves, P.: On Empirical Mode Decomposition and Its Applications. In: IEEE-EURASIP Workshop on Nonlinear Signal and Image Processing NSIP03. Grado(I) (2003) 
12. Wu, Z., Huang, N.E.: Ensemble Empirical Mode Decomposition: A Noise-Assisted Data Analysis Method. Centre for Ocean-Land-Atmosphere Studies, Technical Report No. 193, 51 (2004a), http: / / www . iges . org/pubs/tech.html

13. Wu, Z., Huang, N.E.: A study of the characteristics of white noise using the empirical mode decomposition method. Proceeding of Royal Society of London 460, 1597-1611 (2004)

14. Cecchetti, S.: Monetary Policy and the Financial Crisis of 2007-2008. CEPR Policy Insight (2008)

15. Wu, Z., Huang, N.E., Long, S.R., Peng, C.K.: On the Trend, Detrending, and Variability of Nonlinear and Nonstationary Time Series. Proceedings of the National Academy of Sciences 104, 1488-1489 (2007)

16. Ferderer, J.P.: Oil price volatility and the macroeconomy. Journal of Macroeconomy 18, $1-26$ (1996) 